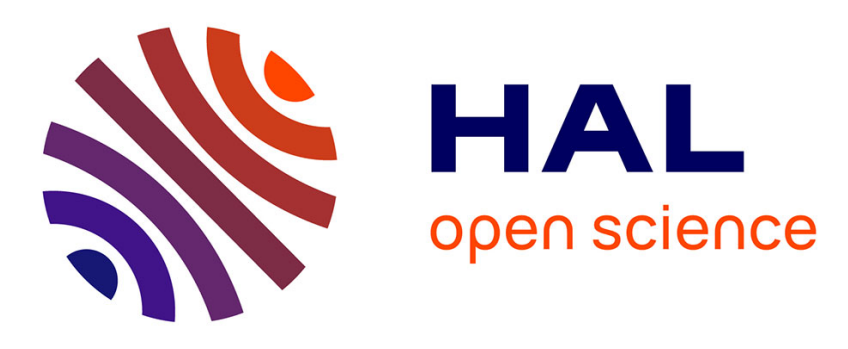

\title{
Bandpass delta sigma optimization using parameter identification
}

\author{
David Gautier, Renaud Lemoine, Francis Huin, Smail Bachir, Claude \\ Duvanaud
}

\section{- To cite this version:}

David Gautier, Renaud Lemoine, Francis Huin, Smail Bachir, Claude Duvanaud. Bandpass delta sigma optimization using parameter identification. TAISA Conference and IEEE Northeast Workshop on Circuits and Systems NEWCAS-TAISA, Jun 2008, Montreal, Canada. pp.209 - 212, 10.1109/NEWCAS.2008.4606358 . hal-00782922

\section{HAL Id: hal-00782922 \\ https://hal.science/hal-00782922}

Submitted on 30 Jan 2013

HAL is a multi-disciplinary open access archive for the deposit and dissemination of scientific research documents, whether they are published or not. The documents may come from teaching and research institutions in France or abroad, or from public or private research centers.
L'archive ouverte pluridisciplinaire HAL, est destinée au dépôt et à la diffusion de documents scientifiques de niveau recherche, publiés ou non, émanant des établissements d'enseignement et de recherche français ou étrangers, des laboratoires publics ou privés. 


\section{BandPass Delta Sigma Optimization Using Parameter Identification}

\author{
David Gautier \\ Renaud Lemoine \\ and Francis Huin \\ ACCO SEMICONDUCTOR \\ 21 bis rue d'hennemont \\ 78100 Saint Germain en Laye , France
}

Email: david.gautier, renaud.lemoine, francis.huin@acco-semi.com

\author{
Smail Bachir \\ and Claude Duvanaud \\ University of Poitiers, LAII \\ 42 av. du Recteur Pineau 86022 Poitiers, France \\ Email: sbachir, cduvanaud@iutang.univ-poitiers.fr
}

\begin{abstract}
In this paper, a new method to optimize the noise shape of a bandpass delta sigma (BPDS) modulator for a digital transmitter is presented. The optimal coefficients of BPDS structure are achieved by minimization of a quadratic criterion based on prediction error. To demonstrate the effectiveness of this approach, simulated results for a $6^{\text {th }}$ order BPDS structure are presented.
\end{abstract}

\section{INTRODUCTION}

Trend in CMOS transceiver is to replace analog blocks by digital ones. Future transmitter could be built based on Sigma Delta modulators (SDM) [1]. One of SDM major properties is its ability to shape the quantization noise [2]. This in turn could create an issue in meeting spurious emission requirements. In current state of the art BPDS modulators, design methods are used to minimize the noise at proximity of the signal frequency, leading to improvement of the Signal to Noise Ratio (SNR) [3]-[4]. The objective of the proposed method is to optimize the shape of quantization noise according to spurious specification defined in particular frequency bandwidths. To demonstrate the interest of the method, it was validated with an usual RF standard, WCDMA FDD: at the top of spurious emission requirements, this standard requires the transmitter to generate a very low noise in the receive channel. Because other elements of the transmitter can modify the Noise Transfer Function (NTF), the frequency response of a generic duplexer is taken in account in the noise shape specification

The proposed method is based on parameter estimation by minimization of quadratic error between an ideal filter and BPDS modulator. Ideal filter will be design using Digital Filter Design from MATLAB/SIMULINK according to the noise shape specification achieved from a generic frequency response of duplexers and the standard. Initialization of the parameter estimation is achieved by a Delta Sigma Toolbox ${ }^{1}$. Performances studied are the Adjacent Channel Leakage power Ratio (ACLR) and the margin with standard (spurious).

\footnotetext{
${ }^{1}$ Delta Sigma Toolbox can be downloaded from MATWORKS web site http://www.mathworks.com/matlabcentral and the function RealizeNTF was use to calculate the initial BPDS coefficients
}

\section{WCDMA AND NOISE SHAPE SPECIFICATION}

Among the WCDMA standard bands, Band 1 was selected as it has many spurious specifications defined in different frequency bands, and a gap of $130 \mathrm{MHz}$ between Transmit (TX) and Receive (RX) bands. WCDMA band 1 spurious specifications close to Tx carrier are detailed on Table I.

TABLE I

WCDMA BAND 1 SPECIFICATIONS

\begin{tabular}{|l|c|c|}
\hline Specifications & \multicolumn{2}{|c|}{ Values } \\
\hline Frequency Carrier (Tx band) & \multicolumn{2}{|c|}{$1920-1980 \mathrm{MHz}$} \\
\hline Output Power & \multicolumn{2}{|c|}{$24 \mathrm{dBm}(+1 /-3 \mathrm{dBm})$} \\
\hline \multirow{2}{*}{ ACLR } & $33 \mathrm{MH}$ & $F_{c} \pm 10 \mathrm{MHz}$ \\
\hline \multirow{4}{*}{ Spurious } & $1 \mathrm{GHz} \leq \mathrm{f} \leq 12.5 \mathrm{GHz}$ & $43 \mathrm{~dB}$ \\
\cline { 2 - 3 } & $1.805 \mathrm{GHz} \leq \mathrm{f} \leq 1.88 \mathrm{GHz}$ & $-30 \mathrm{dBm} / 1 \mathrm{MHz}$ \\
\cline { 2 - 3 } & $1.8449 \mathrm{GHz} \leq \mathrm{f} \leq 1.8799 \mathrm{GHz} / 100 \mathrm{kHz}$ & $-60 \mathrm{dBm} / 3.84 \mathrm{MHz}$ \\
\cline { 2 - 3 } & $1.8845 \mathrm{GHz} \leq \mathrm{f} \leq 1.9196 \mathrm{GHz}$ & $-41 \mathrm{dBm} / 300 \mathrm{kHz}$ \\
\cline { 2 - 3 } & $2.11 \mathrm{GHz} \leq \mathrm{f} \leq 2.17 \mathrm{GHz}$ & $-60 \mathrm{dBm} / 3.84 \mathrm{MkHz}$ \\
\hline
\end{tabular}

Fig. 1-a displays WCDMA band 1 emission restrictions according to Table I. Resolution bandwidth has been recalculated in $\mathrm{dBm} / \mathrm{Hz}$ to achieve a continuity in the specification. Then the overall transfer function depends on frequency response of both amplifier and duplexers.

A BPDS modulators can be used in a transmitter as described in [1]-[5] and shown on Fig. 2.

Commercially available duplexers, implemented for WCDMA band 1, have similar frequency responses. To demonstrate the flexibility and power of the approach, a generic frequency response has been used, as represented in Fig. 1-b.

From the WCDMA standard spurious requirements and generic frequency response, it results a noise shape specification for the BPDS. The resulting curve is shown in Fig. 1-c. The noise rejection must be maximum in two frequency bands (1.8-1.88GHz and 2.1-2.17GHz (Rx band)).

According to the noise rejection, BPDS must have at least three notches. They will be placed in order to maximized the noise rejection especially in the $\mathrm{Rx}$ band. 

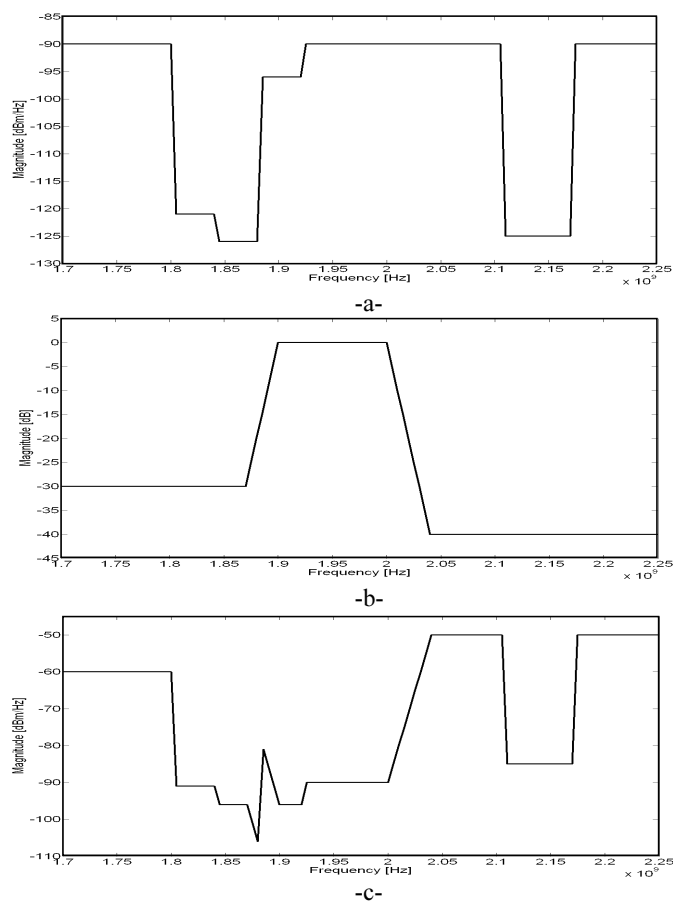

Fig. 1. Noise shape specification : -a- WCDMA band 1; -b- Generic duplexer frequency response for WCDMA band 1; -c- Noise rejection target

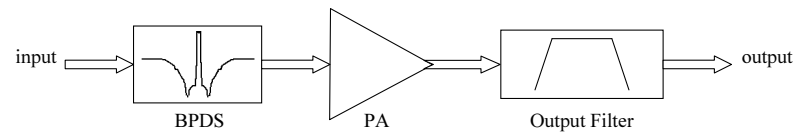

Fig. 2. Diagram of simulated architecture

\section{BPDS MODEL}

\section{A. structure}

A discrete time BPDS design requires to choose different elements like discrete resonator cell $\left(z^{-1}\right.$ or $\left.z^{-2}\right)$, structure (Butterworth or Chebyshev) and form (Cascade-ofIntegrators, FeedBack/FeedForward (CIFB/F) or Cascade-ofResonators, FeedBack/FeedForward (CRFB/F)) [2]. For the proposed study, $z^{-1}$ delay cell combined with Chebyshev structure under CRFB form has been chosen to allow a frequency asymmetric repartition of the notches in the noise shape. Finally, a $6^{t} h$ order BPDS is choosen to achieve the required three notches, as illustrated in Fig. 3.

Input signal is modulated at frequency carrier $\left(F_{c}\right)$ and BPDS is sampled at four times $F_{s}=4 . F_{c}$.

\section{B. State space representation}

BPDS is defined by two transfer functions, the Signal Transfer Function (STF) and the Noise Transfer Function (NTF). In our case, the NTF function is used in optimization to extract the BPDS feedback parameters noted $a_{i}$ and notches coefficients noted $g_{i}$ as referenced on Fig. 3. The CRFB structure is described in state space model. This representation based on transition matrix allows to describe easily the STF and the NTF function. For a $6^{\text {th }}$ order $\mathrm{BPDS}^{2}$, the state space model is defined by the following equations :

$$
\left\{\begin{array}{l}
\underline{x}_{n+1}=A \underline{x}_{n}+B \underline{e}_{n} \\
y_{n}=C^{T} \cdot \underline{x}_{n}
\end{array}\right.
$$

where

$$
\begin{aligned}
A & =\left[\begin{array}{cccccc}
1 & -g_{1} & 0 & 0 & 0 & 0 \\
1 & 1-g_{1} & 0 & 0 & 0 & 0 \\
0 & 1 & 1 & -g_{2} & 0 & 0 \\
0 & 1 & 1 & 1-g_{2} & 0 & 0 \\
0 & 0 & 0 & 1 & 1 & -g_{3} \\
0 & 0 & 0 & 1 & 1 & 1-g_{3}
\end{array}\right] \\
B & =\left[\begin{array}{cc}
1 & -a_{1} \\
1 & -a_{1}-a_{2} \\
0 & -a_{3} \\
0 & -a_{3}-a_{4} \\
0 & -a_{1} \\
0 & -a_{1}-a_{1}
\end{array}\right], C=\left[\begin{array}{l}
0 \\
0 \\
0 \\
0 \\
0 \\
1
\end{array}\right]
\end{aligned}
$$

$\underline{x}_{n}^{T}=\left[\begin{array}{llll}x_{1} & x_{2} & \cdots & x_{6}\end{array}\right]:$ transposed state space vector

$\underline{e}_{n}=\left[\begin{array}{l}u_{n} \\ v_{n}\end{array}\right]:$ input vector

The state space diagram is represented in Fig. 4. Using this representation, the discrete STF and NTF functions can be achieved according to:

$$
\left[\begin{array}{ll}
S T F & N T F
\end{array}\right]=C^{T}\left(z I-A^{\prime}\right)^{-1} B+D
$$

where $A^{\prime}=A+B \cdot\left[\begin{array}{lll}0 & \cdots & 1\end{array}\right]$ and $D=\left[\begin{array}{ll}0 & 1\end{array}\right]$

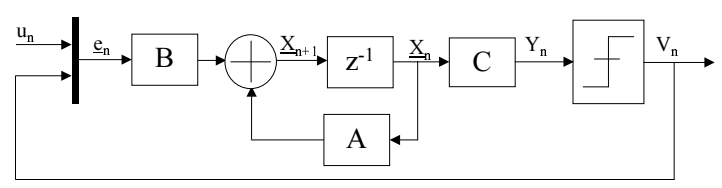

Fig. 4. Block diagram of corresponding state space representation

\section{PARAMETER IDENTIFICATION ALGORITHM}

Parameter estimation is the procedure which allows the determination of the mathematical representation of a real system from experimental data [6]. The block diagram of parameter identification with Output Error technique is shown in Fig. 5. This technique is based on minimization of quadratic error in time domain between desired discrete filter and NTF function of BPDS.

For the case of $6^{\text {th }}$ BPDS optimization, the previous state space model is considered (1) and the following parameter vector is defined :

$$
\underline{\theta}=\left[\begin{array}{lllllll}
a_{1} & a_{2} & \cdots & a_{6} & g_{1} & g_{2} & g_{3}
\end{array}\right]^{T}
$$

The identification problem is to estimate the values of the parameters $\underline{\theta}$ using $K$ samples of the desired filter output $\left(y_{n}\right)$ which is excited by $K$ samples of a white noise uniformly distributed over $[-1,1]\left(v_{n}\right)$ [8]. Thus, the output prediction error is defined as follow:

\footnotetext{
${ }^{2}$ The proposed state space model can be generalized to an $\mathrm{n}^{\text {th }}$ order
} 


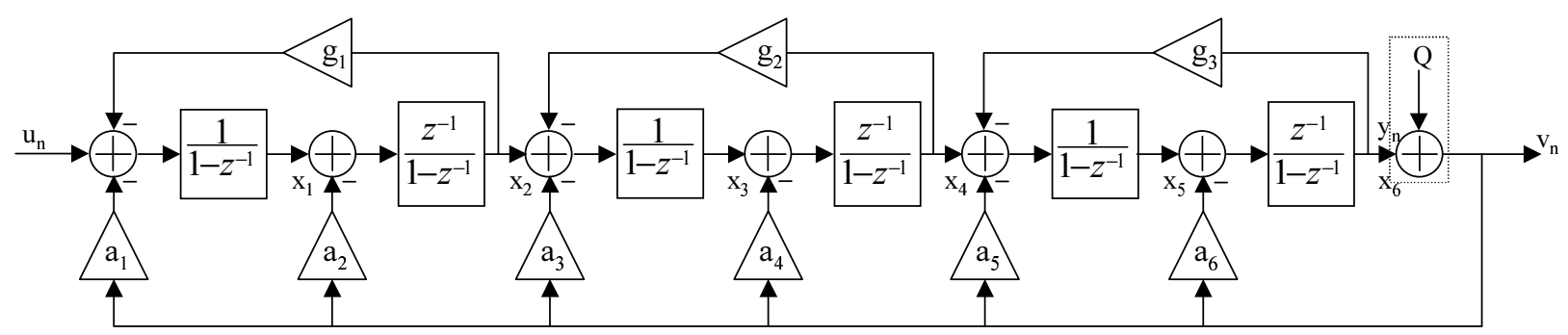

Fig. 3. The $6^{\text {th }}$ order BPDS modulator with CRFB structure

$$
\varepsilon_{n}=y_{n}-\hat{y}_{n}(\underline{\hat{\theta}}, v)
$$

where the estimated NTF output $\hat{y}_{n}$ is obtained by simulations of the NTF (2) and $\underline{\hat{\theta}}$ is an estimation of optimal parameter vector $\underline{\theta}$ (best $a_{i}$ and $g_{i}$ ).

As a general rule, parameter estimation with Output Error technique is based on minimization of a quadratic criterion defined as:

$$
J=\sum_{n=1}^{K} \varepsilon_{n}^{2}=\sum_{n=1}^{K}\left(y_{n}-\hat{y}_{n}\right)^{2}
$$

Optimal values of $\underline{\theta}$ are achieved by Non Linear Programming (NLP) methods. Practically, Marquardt's algorithm [7] is used for off-line estimation:

$$
\underline{\hat{\theta}}_{i+1}=\underline{\hat{\theta}}_{i}-\left\{\left[J_{\theta \theta}^{\prime \prime}+\lambda \cdot I\right]^{-1} \cdot J_{\theta}^{\prime}\right\}_{\underline{\hat{\theta}}=\underline{\theta}_{i}}
$$

with

$J_{\theta}^{\prime}=-2 \cdot \sum_{n=1}^{K} \underline{\varepsilon}_{n}^{T} \cdot \underline{\sigma}_{n, \underline{\theta}}:$ gradient.

$J_{\theta \theta}^{\prime \prime} \approx 2 \cdot \sum_{n=1}^{K} \underline{\sigma}_{n, \underline{\theta}} \cdot \underline{\sigma}_{n, \underline{\theta}}^{T}:$ hessian.

$\lambda$ : monitoring parameter.

$\underline{\sigma}_{n, \underline{\theta}}=\frac{\partial \hat{\underline{y}}}{\partial \underline{\theta}}$ : output sensitivity function.

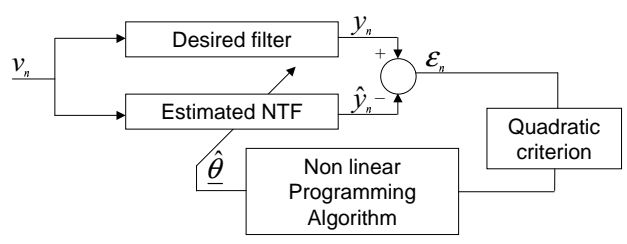

Fig. 5. BPDS optimization scheme

\section{Simulation RESUlTS}

\section{A. Digital filter design}

First, a discrete Chebyshev StopBand filter (NTF form) is designed according to the noise shape specification shown in Fig. 1-c. Filter coefficients are tuned in order to have an important noise rejection in frequency ranges defined in section II.

Notches positions are defined by poles placement in the unit disk to satisfy the stability condition. However, the noise rejection form of the desired NTF is obtained with the zeros placement. During simulation, the filter is excited by the white noise previously defined in section IV. The coefficients of this digital filter and its simulation results in time-domain will be used in optimization procedure.

\section{B. Estimation results}

The proposed parameter identification method is used to adjust the BPDS coefficients. Appropriated initial values, noted $\underline{\theta}_{\text {init }}$, are required to assure convergence of the identification procedure. For example, $\underline{\theta}_{\text {init }}$ can be achieved using Delta Sigma Toolbox. Initial coefficients are listed in Table II and the resulting NTF is presented on Fig. 7, noted Initial NTF. In this case the NTF correspond to a important reduction of noise around the carrier frequency.

At the end of the proposed identification procedure, a new set of coefficients $(\underline{\hat{\theta}})$ is achieved. Table II shows $\underline{\hat{\theta}}$. The resulting BPDS parameters allow closed performances between NTF and ideal filter, as illustrated by time or frequency domain simulations.

TABLE II

INITIAL AND OPTIMIZED COEFFICIENTS

\begin{tabular}{|l|c|c|c|c|c|c|c|}
\hline \multicolumn{2}{|c|}{ index (i) } & 1 & 2 & 3 & 4 & 5 & 6 \\
\hline \hline \multirow{2}{*}{$\dot{\theta}_{\text {init }}$} & $a_{i}$ & 0.042811 & -0.043716 & -0.246777 & 0.002641 & -0.555591 & 0.555591 \\
\cline { 2 - 8 } & $g_{i}$ & 1.9952 & 2 & 2.0047 & - & - & - \\
\hline \hline \multirow{2}{*}{$\hat{\theta}$} & $a_{i}$ & -0.030688 & -0.086103 & -0.357849 & -0.00282 & -0.461228 & 0.602107 \\
\cline { 2 - 8 } & $g_{i}$ & 1.7941 & 2.2532 & 1.9524 & - & - & - \\
\hline
\end{tabular}

Fig. 6-a illustrates simulation results in Time-domain of the output desired filter and the estimated NTF. Fig. 6-b shows quadratic error $\left(\varepsilon_{n}\right)$ and confirms that the BPDS coefficients calculated are in agreement with the response of the desired filter.

Fig. 7 shows simulated NTF at the BPDS output with initialization coefficients, optimized coefficients and WCDMA constraints. The result clearly shows that the NTF is optimized to satisfy the target standard unlike NTF corresponding to initial values which is optimized for Signal to Noise Ratio ( SNR).

For more evaluation, simulations are done with ADVANCED DESIGN SYSTEM (ADS software) using an input signal with a frequency carrier of $F_{c}=1.98 \mathrm{GHz}$ and a power of $-26 \mathrm{dBm}$, sampling frequency of $F_{s}=7.92 \mathrm{GHz}$ and a commercial duplexer. Fig. 8 shows the filtered BPDS output spectrum for different bandwidth resolutions and the corresponding spurious band for WCDMA band 1 standard. 

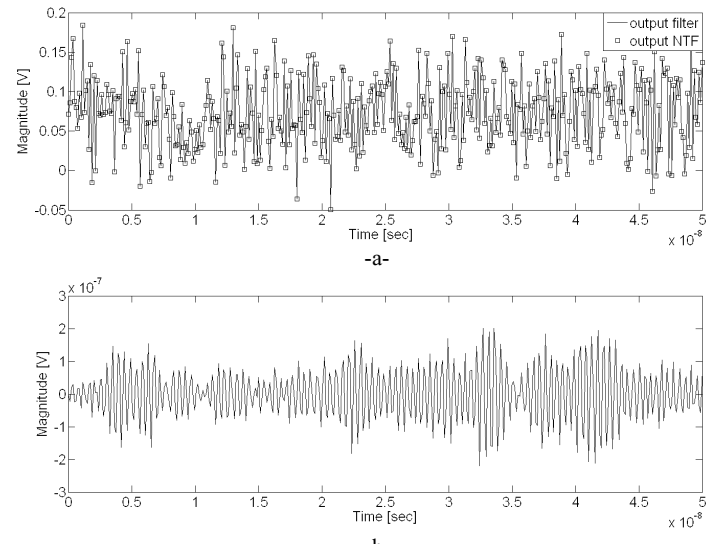

Fig. 6. Time-domain simulation

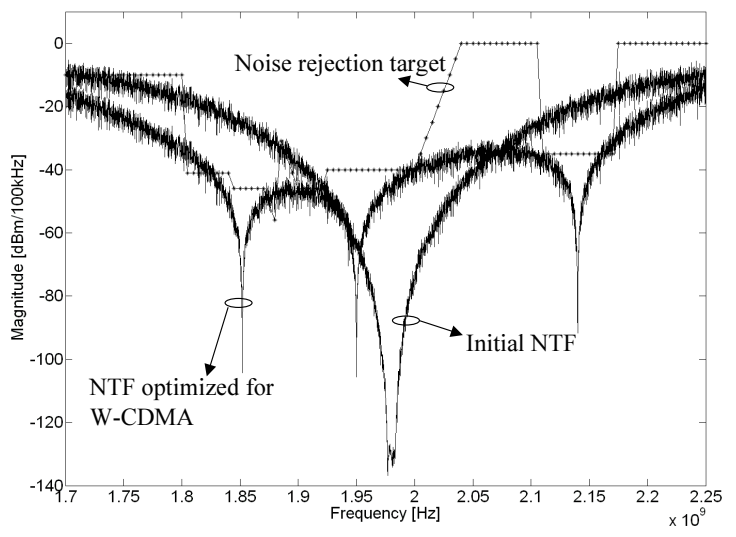

Fig. 7. Frequency-domain simulation

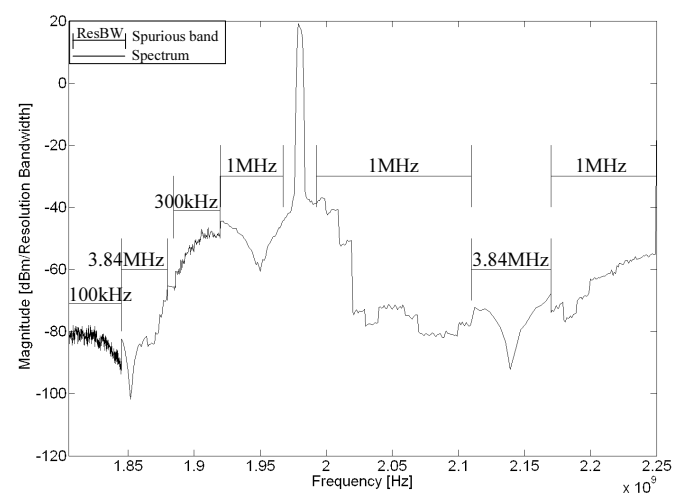

Fig. 8. Output filtered with different resolution bandwidth
As can be seen, the BPDS spectrum has been correctly shaped and would have $10 \mathrm{~dB}$ margin with the spurious emission requirements. However we can notice that the simulated structure would still generate significant amount of noise in the receive band. Output power in the channel equal $24 \mathrm{dBm}$. ACLR results given in Table III, demonstrated that the ACLR specifications are achieved while the SNR in the Tx band has been reduced.

TABLE III

ACLR VALUES

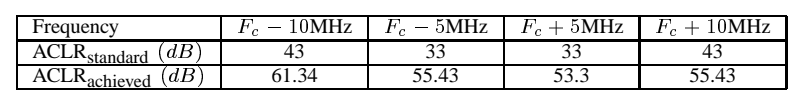

\section{CONCLUSION}

A new method for BPDS modulators optimization has been developed. The procedure is based on output error approach allowing the parameters estimation according to quadratic criterion. The BPDS feedback and notches parameters are iteratively corrected to satisfy the spurious and ACLR of the WCDMA band 1 standard. The proposed optimization method allows to take into account the frequency response of transmitter components, like the output filter. The method can be implemented for analog/digital, Lowpass/Bandpass and generalized to $\mathrm{n}^{\text {th }}$ modulators order.

\section{ACKNOWLEDGMENT}

The authors would like to thank ACCO development and research division for their contribution in this study. They also whish to thank Mr. Jean Louis Dornstetter for the directions he provided as well as for is fruitful discussions.

\section{REFERENCES}

[1] J. Ketola, J. Sommarek, J. Vankka and K. Halonen, "Transmitter utilising Bandpass Delta-Sigma modulator and switching mode power amplifier" IEEE Circuits and Systems, Proceedings of the 2004 Internationnal Symposium, vol 1, pp. I-633-636, May 2004.

[2] R. Schreier and Gabor C. Temes, "Understanding delta sigma data converters" book published by John Wiley \& Sons Inc., 2005.

[3] S. Jantzi, R. Schreier and M. Snelgrove, "Bandpass Sigma-Delta Analogto-Digital Conversion" IEEE Circuits and Systems, vol 38, no. 11, pp. 1406-1409, Nov 1991.

[4] J. Rode, J. Hinrichs and P. Asbeck, "Transmitter Architecture Using Digital Generation of RF Signals"IEEE Radio and Wireless Conference, pp. 245-248, August 2003.

[5] M. Iwamoto, A. Jayaraman, G. Hanington, P.F. Chen, A. Bellora, W. Thornton, L.E. Larson and P.M. Asbeck, "Bandpass delta-sigma classS amplifier" ELECTRONICS LETTERS, vol. 36, no. 12, pp. 1010-1012, June 2000.

[6] L. Ljung, System identification: Theory for the user, Prentice Hall, USA, 1987.

[7] D. W. Marquardt, An Algorithm for least-squares estimation of non-linear parameters, Soc. Indust. Appl. Math, VOL. 11, NO. 2, pp. 431-441, 1963.

[8] Kirk C.-H. Chao, Shujaat Nadeem, Wai L. Lee And Charles G. Sodini, "A Higher Order Topology for Interpolative Modulators for Oversampling A/D Converters" IEEE Transactions on circuits and systems, vol 32, no. 3, pp. 309-318, March 1990. 